\title{
Controlling the interactions of a few cold Rb Rydberg atoms by radio-frequency-assisted Förster resonances
}

\author{
D. B. Tretyakov ${ }^{1}$, V. M. Entin ${ }^{1}$, E. A. Yakshina ${ }^{1,2,3}$, I. I. Beterov ${ }^{1,2}$, C. Andreeva ${ }^{4,5}$, and I. I. Ryabtsev ${ }^{1,2,2}$ 决 \\ ${ }^{1}$ Rzhanov Institute of Semiconductor Physics SB RAS, 630090 Novosibirsk, Russia \\ ${ }^{2}$ Novosibirsk State University, 630090 Novosibirsk, Russia \\ ${ }^{3}$ Russian Quantum Center, Skolkovo, Moscow Region, 143025, Russia \\ ${ }^{4}$ University of Latvia, LV-1002 Riga, Latvia and \\ ${ }^{5}$ Institute of Electronics, Bulgarian Academy of Sciences, Sofia 1784, Bulgaria
}

(Dated: 31 October 2014)

\begin{abstract}
Long-range interactions between cold Rydberg atoms, which are used in many important applications, can be enhanced using Förster resonances between collective many-body states controlled by an external electric field. Here we report on the first experimental observation of highly-resolved radio-frequency-assisted Förster resonances in a few cold Rb Rydberg atoms. We also observed radio-frequency-induced Förster resonances which cannot be tuned by a dc electric field. They imply an efficient transition from van der Waals to resonant dipole-dipole interaction due to Floquet sidebands of Rydberg levels appearing in the rf-field. This method can be applied to enhance the interactions of almost arbitrary Rydberg atoms with large principal quantum numbers.

PACS numbers: $32.80 . \mathrm{Ee}, 34.10 .+\mathrm{x}, 32.70 . \mathrm{Jz}, 32.30 . \mathrm{Bv}, 32.80 . \mathrm{Rm}$
\end{abstract}

Long-range interactions between highly-excited Rydberg atoms are being investigated for several important applications like neutral-atom quantum computing [1], quantum simulations [2], phase transitions in cold Rydberg gases [3], or nonlinear optics with single photons [4]. Depending on the particular Rydberg states, these are van der Waals (vdW) or dipole-dipole (DD) interactions with different dependences on interatomic distance $R$ ( $R^{-6}$ and $R^{-3}$, correspondingly).

Atoms in an identical $n L$ Rydberg state generally interact via vdW, which is much weaker than DD at long distances (longer than the Rydberg atom size that scales as $n^{2}$ ). To make identical atoms interact via $\mathrm{DD}$, the Rydberg state should be tuned exactly midway between two other Rydberg states of the opposite parity to induce a Förster resonance [5]. It can be tuned using the Stark effect in a dc electric field. This method, however, works only for a limited number of Rydberg states. For example, in Rb atoms, narrow Stark-tuned Förster resonances between neighboring Rydberg states can be obtained for $n P_{3 / 2}$ states with $n \leq 38$ [6-8], $n D_{3 / 2}$ states with $n \geq 40$, and $n D_{5 / 2}$ states with $n \geq 43$ [9-11].

Other methods are thus required to control the interactions and tune vdW to DD for arbitrary Rydberg states. One such method was demonstrated in Refs. [9], where the energies of Rb Rydberg states were adjusted by an ac Stark shift in a strong nonresonant microwave field at $28.5 \mathrm{GHz}$ or in a nearly resonant to a Rydberg transition microwave field at $1.356 \mathrm{GHz}$. Another method is to apply a resonant microwave field that drives a transition between Rydberg states of the opposite parity and mixes them up [12-14].

It is also possible to control Rydberg interactions by

*Electronic address: ryabtsev@isp.nsc.ru microwave-assisted Förster resonances [15-19], when one or several microwave photons compensate for the energy defect and induce transitions between the initial and final many-body collective states of the Förster resonance. Microwave or even radio-frequency (rf) photons can provide the tunability of Förster resonances in a wide range.

In this Rapid Communication we demonstrate that rf electric fields can be used to induce "inaccessible" Förster resonances, which cannot be tuned by a dc electric field, and that it leads to an efficient transition from vdW to DD interaction for a few cold Rb Rydberg atoms. A new point compared to Refs. $[9,12-14]$ is that the rf-field drives the transitions not between Rydberg states of a single atom (typical frequencies 10-100 GHz) but between nearly degenerate collective states of the quasi-molecule formed by the interacting Rydberg atoms (typical frequencies $10-100 \mathrm{MHz}$ ).

The process under study is the Förster resonant energy transfer $\mathrm{Rb}\left(n P_{3 / 2}\right)+\mathrm{Rb}\left(n P_{3 / 2}\right) \rightarrow \operatorname{Rb}\left(n S_{1 / 2}\right)+$ $\operatorname{Rb}\left([n+1] S_{1 / 2}\right)$ due to the dipole-dipole interaction of two or more cold Rb Rydberg atoms in a small laser excitation volume $[8,20,21]$. The energy detuning of this resonance $\hbar \Delta=E\left(n S_{1 / 2}\right)+E\left([n+1] S_{1 / 2}\right)-2 E\left(n P_{3 / 2}\right)$ is controlled by a weak dc electric field. It can be tuned to zero for Rydberg states with $n \leq 38$ as shown in Fig. 1(a) for the $37 P_{3 / 2}$ state, while for states with $n \geq 39$ the dc electric field increases $\Delta$ and the resonance could be induced only by the rf-field [see Fig. 2 (a) for the $39 P_{3 / 2}$ state].

Experiments were performed with cold ${ }^{85} \mathrm{Rb}$ atoms in a magneto-optical trap. The excitation to the $n P_{3 / 2}\left(\left|\mathrm{M}_{J}\right|=1 / 2\right)$ Rydberg state is realized via threephoton transition $5 S_{1 / 2} \rightarrow 5 P_{3 / 2} \rightarrow 6 S_{1 / 2} \rightarrow n P_{3 / 2}$ by means of three $\mathrm{cw}$ lasers modulated to form $2 \mu$ s exciting pulses at a repetition rate of $5 \mathrm{kHz}$ [22]. The small Rydberg excitation volume of $30-40 \mu \mathrm{m}$ size is formed using crossed-beam geometry [20]. 
Our experiment provides atom-number-resolved measurement of the signals obtained from $N=1-5$ of the detected Rydberg atoms with a detection efficiency of $65 \%$ [8]. It is based on selective field ionization (SFI) detector with channel electron multiplier and post-selection technique [23]. The normalized $N$-atom signals $S_{N}$ are the average fractions of atoms that have undergone a transition to the final $n S$ state. In fact, the signals measured in our experiment correspond to the detection of $N$-body collective states, which are used in theoretical calculations $[8,21]$. Compared to the previous works on rf-assisted Förster resonances $[17,18]$ we deal with a few Rydberg atoms in an identical $n P_{3 / 2}$ state, interacting in a single small excitation volume.

We use Stark-switching technique $[7,11]$ to switch the interactions on and off as depicted in Fig. 1(b). The laser excitation occurs for $2 \mu$ s at a fixed electric field of $5.6 \mathrm{~V} / \mathrm{cm}$. Then the field decreases to a lower value near the resonant electric field $\left(1.79 \mathrm{~V} / \mathrm{cm}\right.$ for the $37 P_{3 / 2}$ state), which acts for $3 \mu$ s until the field increases back to $5.6 \mathrm{~V} / \mathrm{cm}$. The lower electric field is slowly scanned across the Förster resonance and SFI signals are accumulated for $10^{3}-10^{4}$ laser pulses. An rf pulse with variable amplitude $(0-300 \mathrm{mV})$ and frequency $(10-100 \mathrm{MHz})$ is superimposed on the lower dc field.

Figures 1(c)-1(f) present the spectra $S_{N}$ of the Förster resonance $\mathrm{Rb}\left(37 P_{3 / 2}\right)+\operatorname{Rb}\left(37 P_{3 / 2}\right) \rightarrow \operatorname{Rb}\left(37 S_{1 / 2}\right)+$ $\mathrm{Rb}\left(38 S_{1 / 2}\right)$ in a $15 \mathrm{MHz}$ rf-field of various amplitudes recorded for $N=2-5$ detected Rydberg atoms as a function of the dc electric field. Without the rf-field in Fig. 1(c), the single narrow peak at $1.79 \mathrm{~V} / \mathrm{cm}$ is the "true" Stark-tuned Förster resonance, whose amplitude and width grow with $N$ according to theory [8,21]. The spectrum $S_{2}$ corresponds to DD interaction of just two Rydberg atoms in the interaction volume [8]. Its width of $16 \mathrm{mV} / \mathrm{cm}$ is equivalent to the frequency width of $1.9 \mathrm{MHz}$.

Application of a $150 \mathrm{mV}$ rf-field [Fig.1(d)] induces additional Förster resonances, which are rf-assisted resonances of various orders, as shown in the scheme in Fig. 1(a) for the energy levels of the initial $37 P+37 P$ and final $37 S+38 S$ collective states in the dc electric field. The arrows indicate rf-induced Förster resonances of different orders. As $N$ increases, the resonance amplitudes grow and the rf-assisted resonances become more pronounced in Fig. 1(d) due to increase in the total DD interaction energy.

The frequency interval between the peaks in Fig. 1(d) corresponds exactly to $15 \mathrm{MHz}$, taking into account the known polarizabilities of these Rydberg states [20]. Figures 1(e)-(f) present such spectra as a function of frequency detuning for 200 and $300 \mathrm{mV}$ rf-amplitudes. The observed high-order resonances have almost the same amplitude and width as the low-order ones if the rfamplitude is large enough. This means that the vdW undergoes a transition to DD with high efficiency, reaching 50-100\%. By changing the rf-frequency we were able to control the positions of the peaks with high precision.
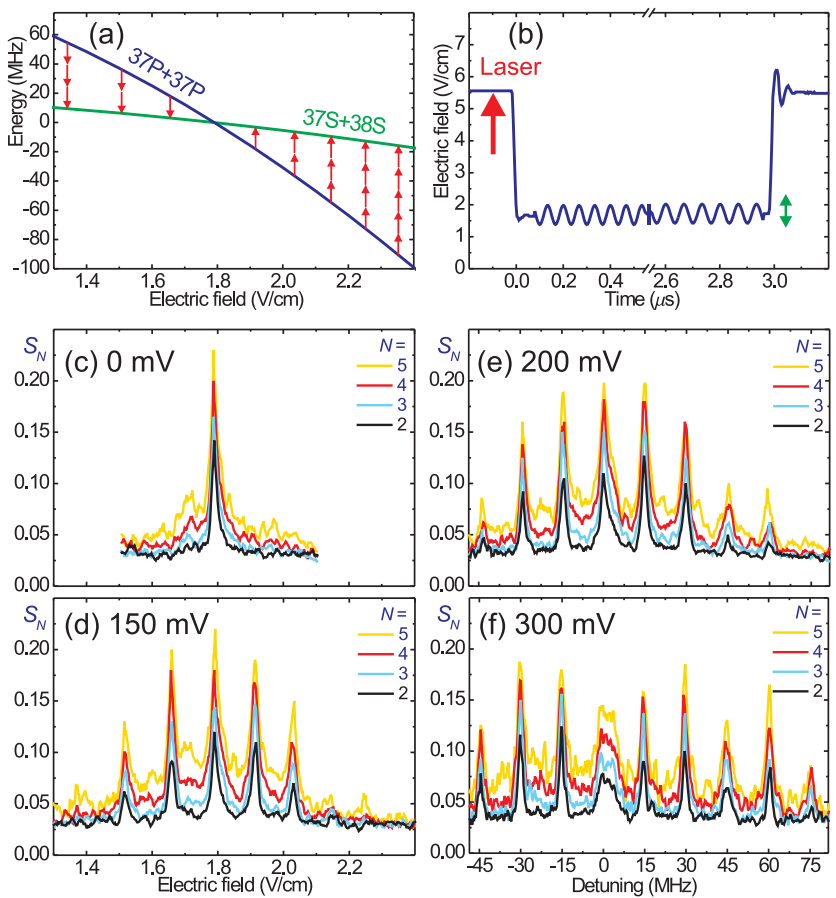

FIG. 1: (color online). (a) Energy levels of the initial $37 P+37 P$ and final $37 S+38 S$ collective states of two interacting Rb Rydberg atoms in an electric field. The arrows indicate rf-induced Förster resonances $\mathrm{Rb}\left(37 P_{3 / 2}\right)+\mathrm{Rb}\left(37 P_{3 / 2}\right) \rightarrow$ $\mathrm{Rb}(37 S)+\mathrm{Rb}(38 S)$ of various orders at rf-frequency $15 \mathrm{MHz}$. (b) Time dependence of the electric field. (c) Single Förster resonance without rf-field for $N=2-5$ detected Rydberg atoms as a function of the electric field. (d) RF-assisted Förster resonances at $150 \mathrm{mV}$ rf-amplitude, for the same scale. (e)-(f) $\mathrm{RF}$-assisted Förster resonances as a function of frequency detuning at 200 and $300 \mathrm{mV}$ rf-amplitudes, respectively.

Now we turn to the "inaccessible" Förster resonances, which cannot be tuned by dc electric field. An example is the Förster resonance $\operatorname{Rb}\left(39 P_{3 / 2}\right)+\mathrm{Rb}\left(39 P_{3 / 2}\right) \rightarrow$ $\operatorname{Rb}\left(39 S_{1 / 2}\right)+\operatorname{Rb}\left(40 S_{1 / 2}\right)$ whose collective energy levels in the dc electric field are shown in Fig. 2(a). The dc field alone increases the energy detuning $\Delta$ and makes the interaction weaker. However, our experience with the Förster resonance for the $37 P_{3 / 2}$ state suggests that the rf-field can induce transitions between collective states, so the Förster resonance occurs irrespective of the possibility of tuning it by the dc field alone. The dc field, however, should be applied to increase the efficiency, as will be discussed below.

Figures 2(b)-2(d) present the experimental records of the Förster resonance $\operatorname{Rb}\left(39 P_{3 / 2}\right)+\operatorname{Rb}\left(39 P_{3 / 2}\right) \rightarrow$ $\mathrm{Rb}\left(39 S_{1 / 2}\right)+\operatorname{Rb}\left(40 S_{1 / 2}\right)$ in the rf-field at frequencies 90, 95, and $100 \mathrm{MHz}$, correspondingly. The rf-induced Förster resonance is clearly seen. Its position depends on the rf-frequency, while its width and height depend on the number of atoms $[8,21]$. For $N=2$ the width is 18 $\mathrm{mV} / \mathrm{cm}$, corresponding to $1.1 \mathrm{MHz}$. At higher frequencies it appears at higher dc electric field. The resonance is 


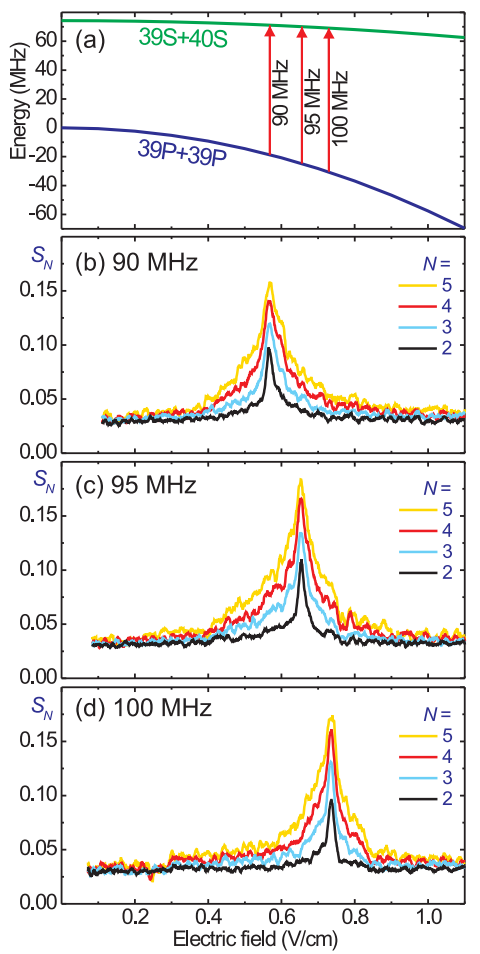

FIG. 2: (color online). (a) Energy levels of the initial $39 P+39 P$ and final $39 S+40 S$ collective states of two Rydberg atoms in the electric field. The dc electric field alone cannot tune a Förster resonance $\mathrm{Rb}\left(39 P_{3 / 2}\right)+\mathrm{Rb}\left(39 P_{3 / 2}\right) \rightarrow$ $\mathrm{Rb}(39 S)+\mathrm{Rb}(40 S)$. The rf-photons couple these collective states and induce resonant dipole-dipole interaction. (b)-(d) $\mathrm{RF}$-assisted Förster resonances for $N=2-5$ detected Rydberg atoms at $100 \mathrm{mV}$ amplitude and 90, 95, and $100 \mathrm{MHz}$ rf-field frequency, respectively.

quite efficient, as its amplitude is comparable with that of the "true" resonance [see Fig. 1(c)] and at $N=5$ it is close to the maximum possible value of 0.25 for a disordered atom ensemble [21]. Figure 2 thus evidences the possibility to tune vdW to DD interaction with high efficiency using the rf-field, which is resonant to a quasi-molecular transition instead of atomic transition.

The physical interpretation of the rf-assisted Förster resonances was given in previous papers $[15,18]$. We will emphasize some important features in the two possible approaches. On the one hand, the rf-field induces transitions between quasi-molecular collective states, as shown in Figs. 1(a) and 2(a). Being absorbed or emitted by a quasi-molecule consisting of a few Rydberg atoms, the few rf-photons of frequency $\omega$ compensate for the energy defect $\Delta$ when it has values multiples of $\omega$.

On the other hand, rf-assisted Förster resonances can also be explained in terms of the Floquet sidebands induced by a periodic perturbation of the Rydberg energy levels by the rf electric field due to the Stark effect $[15,18]$. Following Ref. [18], one should consider the Stark effect in a composite electric field consisting of dc and rf parts
$F=F_{d c}+F_{r f} \cos (\omega t)$. The energy shift of a Rydberg level with nonzero quantum defect is quadratic and is given by its polarizability $E_{n L}=-\alpha_{n L} F^{2} / 2$. This formula yields

$$
\begin{aligned}
& E_{n L}=-\frac{1}{2} \alpha_{n L}\left[F_{d c}^{2}+\frac{1}{2} F_{r f}^{2}+\right. \\
& \left.2 F_{d c} F_{r f} \cos (\omega t)+\frac{1}{2} F_{r f}^{2} \cos (2 \omega t)\right] .
\end{aligned}
$$

The term $F_{r f}^{2} / 2$ in the brackets is responsible for the ac Stark shift of the Rydberg level in the rf-field [18]. The terms with $\cos (\omega t)$ and $\cos (2 \omega t)$ drive the transitions between collective states as soon as the resonance condition $\Delta=m \omega$ is satisfied, with $m$ being an integer. This can be understood if we use a Floquet approach to find the eigenenergies of a Rydberg atom in dc+rf field [18]. It gives an infinite number of energy sidebands separated by $\omega$ and with relative amplitudes of the wave functions $a_{n L, m}$ described by the generalized Bessel functions

$$
a_{n L, m}=\sum_{k=-\infty}^{\infty} J_{m-2 k}\left(\frac{\alpha_{n L} F_{d c} F_{r f}}{\omega}\right) J_{k}\left(\frac{\alpha_{n L} F_{r f}^{2}}{8 \omega}\right) .
$$

The rf-assisted Förster resonances in this picture arise for the Floquet sidebands that satisfy the resonance condition $\Delta=m \omega$ and intersect at some particular values of the dc electric field. At $F_{d c}=0$ the odd sidebands disappear according to Eq. (2) since only $J_{0}(0)=1$ is nonzero, while the even sidebands are weak. Therefore, the rf-field alone hardly drives the transitions between quasi-molecular collective states with a quadratic Stark effect, so a dc field should also be present.

Figure 3(a) shows the energy levels of the initial $37 P+37 P$ and final $37 S+38 S$ collective states of two Rydberg atoms in electric field in the presence of the first Floquet sidebands at $\pm 15 \mathrm{MHz}$. The red (gray) circles indicate the intersections of the Floquet sidebands, corresponding to rf-assisted Förster resonances. These resonances are clearly seen in Fig. 3(b) on the experimental record at a $100 \mathrm{mV}$ rf-amplitude. Figures 3(c) and $3(\mathrm{~d})$ show the same for the Förster resonance on the $39 P_{3 / 2}$ state at $95 \mathrm{MHz}$ and $100 \mathrm{mV}$. There are much less Floquet intersections for this "inaccessible" Förster resonance, but a narrow second-order resonance at $1.55 \mathrm{~V} / \mathrm{cm}$ is well seen along with a much stronger first-order resonance at $0.66 \mathrm{~V} / \mathrm{cm}$. The first-order resonance saturates and broadens as $N$ increases, while the second-order resonance is unsaturated and remains narrow for all $N$.

Below we discuss the impact of our experimental observations on the studies and applications of long-range interactions between Rydberg atoms. Radio-frequencyassisted "inaccessible" Förster resonances provide a way to tune vdW to resonant DD interactions and increase the interaction strength at long distances. The transition from vdW to DD can be analyzed by the formula 

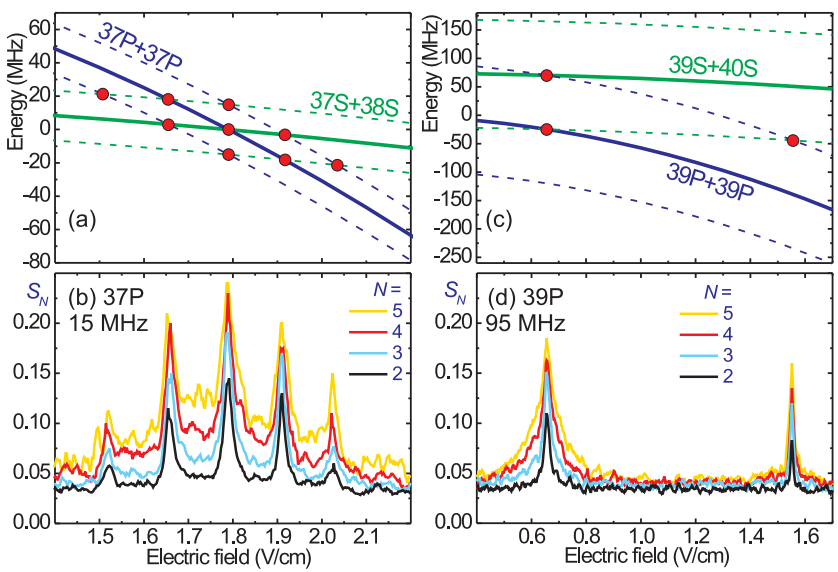

FIG. 3: (color online). (a) Energy levels of the initial $37 P+37 P$ and final $37 S+38 S$ collective states of two Rydberg atoms in the electric field in the presence of the first Floquet sidebands at $\pm 15 \mathrm{MHz}$. The red (gray) circles indicate the intersections of the Floquet sidebands corresponding to rf-assisted Förster resonances. (b) Experimental record of the rf-assisted Förster resonances at a $100 \mathrm{mV}$ rf-amplitude for $N=2-5$ detected Rydberg atoms. (c)-(d) The same for the "inaccessible" Förster resonances on the $39 P$ state at $95 \mathrm{MHz}$ and $100 \mathrm{mV}$. The first- and second-order resonances are observed.

describing the energy shift $\delta E_{P P}$ of the collective $n P+n P$ state at the DD matrix element $V$ from $n P+n P$ state to $n S+(n+1) S$ state and Förster detuning $\Delta$ :

$$
\delta E_{P P}= \pm\left(\sqrt{\frac{\Delta^{2}}{4}+2 V^{2}}-\frac{|\Delta|}{2}\right) .
$$

Here the sign is positive if the $n P+n P$ state lies above the $n S+(n+1) S$ state (this is the case for $n \leq 38$ ), and vice versa. At $\Delta=0$ the interaction is purely $\mathrm{DD}$, while at large detuning it is the vdW with energy shift $\pm 2 V^{2} / \Delta=C_{6} / R^{6}$, where $C_{6}$ is vdW coefficient and $R$ is interatomic distance. The resonant rf-field with $\omega=\Delta / m$ compensates for $\Delta$ and tunes $\mathrm{vdW}$ to DD as if the $\Delta=0$ condition is satisfied.

This can be particularly useful for enhancing the dipole blockade effect in mesoscopic Rydberg ensembles [24]. For example, in a zero electric field the orientation-averaged energy of the $\mathrm{vdW}$ interaction between $\operatorname{Rb}\left(70 P_{3 / 2}\right)$ atoms at $\Delta /(2 \pi)=213 \mathrm{MHz}$ and distance $10 \mu \mathrm{m}$ is estimated to be $1.5 \mathrm{MHz}$, using $C_{6} \approx$ $-1500 \mathrm{GHz} / \mu \mathrm{m}^{6}$ from Ref. [25]. By applying $\sim 0.1 \mathrm{~V} / \mathrm{cm}$ dc electric field and $\sim 0.05 \mathrm{~V} / \mathrm{cm}$ rf-field at $250-280 \mathrm{MHz}$, it can be tuned to DD interaction with energy corresponding to $18 \mathrm{MHz}$, an order of magnitude larger than the vdW energy. For $\mathrm{Rb} n P_{3 / 2}$ states with $n=40-100$ the required rf-frequencies lie in the $100-325 \mathrm{MHz}$ range, and for $n S$ states with $n=70-120$ they are in the 140 $700 \mathrm{MHz}$ range. These are reasonably low rf-frequencies, which can also be found in other alkali-metal atoms [26].

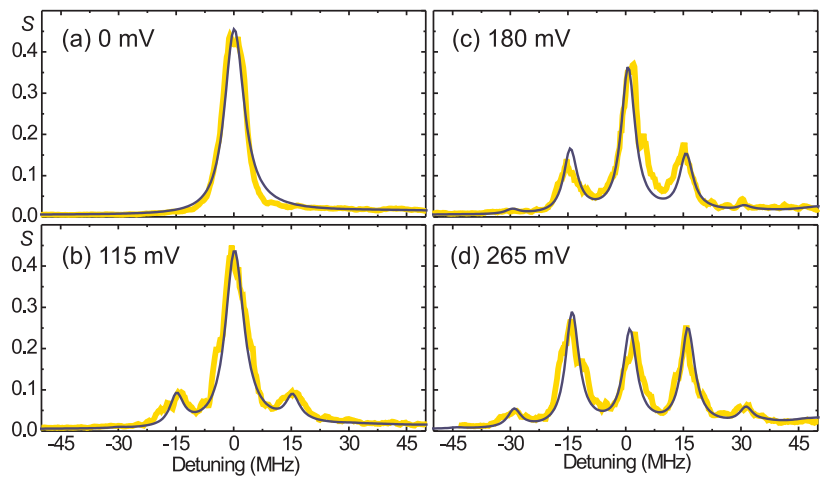

FIG. 4: (color online). Floquet sidebands observed at threephoton laser excitation of the $37 P_{3 / 2}$ state in dc electric field of $1.8 \mathrm{~V} / \mathrm{cm}$ with an admixed rf field at frequency $15 \mathrm{MHz}$ for various rf-amplitudes: (a) $0 \mathrm{mV}$, (b) $115 \mathrm{mV}$, (c) $180 \mathrm{mV}$, (d) $265 \mathrm{mV}$. The yellow (gray) curves are experiment, and blue (dark) curves are theory calculated with our four-level density-matrix model [22].

Radio-frequency-assisted Förster resonances thus significantly extend the range of the Rydberg states suitable for long-range resonant DD interaction.

In our present experiment we first excited Rydberg atoms without the rf-field and then switched it on adiabatically [Fig. 1(b)]. In the experiments on dipole blockade, however, one needs the rf-field to be applied together with the laser excitation. Therefore, the laser radiation should be tuned in resonance not with the unperturbed Rydberg level, but with one of its Floquet sidebands that satisfies the Förster resonance condition $\Delta=m \omega$. We have observed these Floquet sidebands experimentally for the $37 P_{3 / 2}$ state at $\omega /(2 \pi)=15 \mathrm{MHz}$ and various rfamplitudes, as shown in Fig. 4. The yellow (gray) curves are experimental, and blue (dark) curves are theoretical ones calculated with our four-level density-matrix model [22]. The sideband amplitudes are similar to those observed in Fig. 1 for the Stark switched excitation. Weaker Floquet sidebands have also been reported at Rydberg excitation in a $\mathrm{Rb}$ vapor cell [27].

To conclude, we have observed and studied the highly resolved rf-assisted Förster resonances between a few cold Rydberg atoms in a small laser excitation volume. These resonances correspond to single- and multiphoton rf-transitions between many-body collective states of a Rydberg quasi-molecule or to intersections of the Floquet sidebands of Rydberg levels appearing in the rf-field. We have shown that they can be induced both for the "accessible" Förster resonances, which are tuned by the dc field, and for those which cannot be tuned and are "inaccessible". The van der Waals interaction of almost arbitrary high Rydberg states can thus be efficiently tuned to a resonant dipole-dipole interaction using the rf-field with frequencies below $1 \mathrm{GHz}$. This enhances the interaction strength and distance and can give rise to a much stronger dipole blockade effect. 
This work was supported by RFBR (Grants No. 13-0200283 and No. 14-02-00680), by the Russian Academy of
Sciences, by the EU FP7 IRSES Project "COLIMA", and by the Russian Quantum Center.
[1] M. Saffman, T. G. Walker, and K. Mølmer, Rev. Mod. Phys. 82, 2313 (2010).

[2] T. Keating, K. Goya, Y. -Y. Jau, G. W. Biedermann, A. J. Landahl, and I. H. Deutsch, Phys. Rev. A 87, 052314 (2013).

[3] F. Cinti, P. Jain, M. Boninsegni, A. Micheli, P. Zoller, and G. Pupillo, Phys. Rev. Lett. 105, 135301 (2010).

[4] J. Honer, R. Löw, H. Weimer, T. Pfau, and H. P. Büchler, Phys. Rev. Lett. 107, 093601 (2011).

[5] K. A. Safinya, J. F. Delpech, F. Gounand, W. Sandner, and T. F. Gallagher, Phys. Rev. Lett. 47, 405 (1981).

[6] A. L. de Oliveira, M. W. Mancini, V. S. Bagnato, and L. G. Marcassa, Phys. Rev. Lett. 90, 143002 (2003).

[7] S. Westermann, T. Amthor, A. L. de Oliveira, J. Deiglmayr, M. Reetz-Lamour, and M. Weidemüller, Eur. Phys. J. D 40, 37 (2006).

[8] I. I. Ryabtsev, D. B. Tretyakov, I. I. Beterov, and V. M. Entin, Phys. Rev. Lett. 104, 073003 (2010).

[9] P. Bohlouli-Zanjani, J. A. Petrus, and J. D. D. Martin, Phys. Rev. Lett. 98, 203005 (2007); J. A. Petrus, P. Bohlouli-Zanjani, and J. D. D. Martin, J. Phys. B 41, 245001 (2008).

[10] A. Reinhard, K. C. Younge, and G. Raithel, Phys. Rev. A 78, 060702(R) (2008).

[11] J. Nipper, J. B. Balewski, A. T. Krupp, B. Butscher, R. Löw, and T. Pfau, Phys. Rev. Lett. 108, 113001 (2012).

[12] K. Afrousheh, P. Bohlouli-Zanjani, D. Vagale, A. Mugford, M. Fedorov, and J. D. D. Martin, Phys. Rev. Lett. 93, 233001 (2004).

[13] M. Tanasittikosol, J. D. Pritchard, D. Maxwell, A. Gauguet, K. J. Weatherill, R. M. Potvliege, and C. S. Adams, J. Phys. B 44, 184020 (2011).

[14] E. Brekke, J. O. Day, and T. G. Walker, Phys. Rev. A 86, 033406 (2012).

[15] P. Pillet, R. Kachru, N. H. Tran, W. W. Smith, and T. F. Gallagher, Phys. Rev. A 36, 1132 (1987).

[16] P. Pillet, D. Comparat, M. Muldrich, T. Vogt, N. Za- hzam, V. M. Akulin, T. F. Gallagher, W. Li, P. Tanner, M. W. Noel, and I. Mourachko, in Decoherence, Entanglement and Information Protection in Complex Quantum Systems, edited by V. M. Akulin et al., Springer, 2005, p.411.

[17] A. Tauschinsky, C. S. E. van Ditzhuijzen, L. D. Noordam, and H. B. van Linden van den Heuvell, Phys. Rev. A 78, 063409 (2008).

[18] C. S. E. van Ditzhuijzen, A. Tauschinsky, and H. B. van Linden van den Heuvell, Phys. Rev. A 80, 063407 (2009).

[19] Y. Yu, H. Park, and T. F. Gallagher, Phys. Rev. Lett. 111, 173001 (2013).

[20] D. B. Tretyakov, I. I. Beterov, V. M. Entin, I. I. Ryabtsev, and P. L. Chapovsky, J. Exper. Theor. Phys. 108, 374 (2009).

[21] I. I. Ryabtsev, D. B. Tretyakov, I. I. Beterov, V. M. Entin, and E. A. Yakshina, Phys. Rev. A 82, 053409 (2010).

[22] V. M. Entin, E. A. Yakshina, D. B. Tretyakov, I. I. Beterov, and I. I. Ryabtsev, J. Exper. Theor. Phys. 116, 721 (2013).

[23] I. I. Ryabtsev, D. B. Tretyakov, I. I. Beterov, and V. M. Entin, Phys. Rev. A 76, 012722 (2007); Erratum: Phys. Rev. A 76, 049902(E) (2007).

[24] M. D. Lukin, M. Fleischhauer, R. Cote, L. M. Duan, D. Jaksch, J. I. Cirac, and P. Zoller, Phys. Rev. Lett. 87, 037901 (2001); D. Comparat and P. Pillet, J. Opt. Soc. Am. B 27, A208 (2010).

[25] T. G. Walker and M. Saffman, Phys. Rev. A 77, 032723 (2008).

[26] J. H. Gurian, P. Cheinet, P. Huillery, A. Fioretti, J. Zhao, P. L. Gould, D. Comparat, and P. Pillet, Phys. Rev. Lett. 108, 023005 (2012).

[27] M. G. Bason, M. Tanasittikosol, A. Sargsyan, A. K. Mohapatra, D. Sarkisyan, R. M. Potvliege, and C. S. Adams, New J. Phys. 12, 065015 (2010). 